\title{
O. Ritz, Les Métaphores naturelles dans le débat sur la Révolution
}

\section{Timothée Léchot}

\section{(2) OpenEdition}

1 Journals

\section{Édition électronique}

URL : http://journals.openedition.org/studifrancesi/10645

DOI : 10.4000/studifrancesi. 10645

ISSN : 2427-5856

Éditeur

Rosenberg \& Sellier

\section{Édition imprimée}

Date de publication : 1 décembre 2017

Pagination : 554-555

ISSN : 0039-2944

\section{Référence électronique}

Timothée Léchot, « O. Ritz, Les Métaphores naturelles dans le débat sur la Révolution », Studi Francesi [En ligne], 183 (LXI | III) | 2017, mis en ligne le 01 février 2018, consulté le 21 janvier 2021. URL : http:// journals.openedition.org/studifrancesi/10645; DOI : https://doi.org/10.4000/studifrancesi.10645

Ce document a été généré automatiquement le 21 janvier 2021.

\section{cc) $(1) \odot$}

Studi Francesi è distribuita con Licenza Creative Commons Attribuzione - Non commerciale - Non opere derivate 4.0 Internazionale. 


\title{
O. Ritz, Les Métaphores naturelles dans le débat sur la Révolution
}

\author{
Timothée Léchot
}

\section{RÉFÉRENCE}

OLIVIER RITZ, Les Métaphores naturelles dans le débat sur la Révolution, Paris, Classiques Garnier, 2016, «L'Europe des Lumières» 48, 371 pp.

1 Les ondées du 14 juillet 1790 sont «les larmes des aristocrates»; «l'électricité des pensées» caractérise l'Assemblée constituante; les idées contagieuses de Marat se répandent en "exhalaisons pestilentielles»; la Terreur a «l'aspect redoutable d'un volcan immense»... Tempête sublime sur le monde politique, comète explicable et récurrente, tremblement de terre inattendu, éruption foudroyante: la Révolution française est décrite, promue, contestée et digérée à l'aide de métaphores naturelles.

2 Convoquant des objets ou des phénomènes naturels, ou encore des processus scientifiques, ces métaphores sont remarquables par leur fréquence et par leur diversité dans les textes de la décennie révolutionnaire, et dans les écrits de l'Empire qui commentent la Révolution a posteriori. Olivier Ritz n'est pas le premier à aborder cette thématique, mais il lui donne une ampleur particulière. Dans son livre, les métaphores naturelles ne sont pas seulement étudiées pour elles-mêmes: elles forment un prisme à travers lequel l'auteur cherche un nouveau point de vue sur de plus vastes questions, comme l'alliance entre la science et le politique, la légitimation du travail de l'historien et, en point de mire, l'affirmation d'une nouvelle définition de la littérature au tournant du siècle. C'est beaucoup pour un seul ouvrage, mais il y a quelque chose de fascinant à redécouvrir de telles problématiques historiques dans la lorgnette d'une figure de style bien précise.

3 Issu d'une thèse de doctorat, le livre suit une structure canonique: nonobstant le chapitre liminaire qui a une valeur introductive, il contient trois parties composées de trois chapitres eux-mêmes subdivisés trois fois. Une telle organisation appelle une 
progression dialectique: l'auteur ne suit pas une ligne chronologique, mais il progresse de citation en citation et d'analyse en analyse en fonction des rapports d'affinité ou de contraste qu'entretiennent les textes de son corpus. Celui-ci est présenté comme un corpus ouvert dans l'introduction; il recoupe toutes les déclinaisons de la «prose d'idées» (p. 19), du pamphlet à la grande fresque historique en passant par l'essai et le roman. Un choix d'œuvres anglaises et allemandes s'ajoute aux sources françaises. À l'égard de la métaphore, Ritz se range sous la bannière théorique de Joëlle GardesTamine: il regarde cette figure comme l'instrument non pas d'une substitution, mais d'une relation entre deux éléments. Par les associations qu'elle permet, la métaphore contribue donc à créer des savoirs et des discours nouveaux, mais aussi des imaginaires historiques comme l'auteur le montre plus loin, à l'égard de la Terreur.

4 La première partie, «Faire la Révolution», expose les fonctions esthétiques et rhétoriques des métaphores naturelles au sein du débat. Ritz nous invite d'abord à la Fête de la Fédération, moins pour brosser le tableau de cette journée mémorablement pluvieuse que pour exposer la richesse du langage métaphorique des commentateurs et l'interpénétration des discours scientifique et politique. Le ton est donné: la métaphore naturelle doit être considérée comme un instrument textuel polyvalent, mobilisé par tous les partis, capable de fixer ou d'interroger la signification des événements. Ces «métaphores vives» (p. 44) servent à émouvoir, pour inspirer l'amour de la Révolution ou, au contraire, pour susciter la peur en invoquant l'image du tremblement de terre, de la tempête ou du volcan. Les deux options ne sont pas exclusives l'une de l'autre, comme le remarque Ritz, puisque la métaphore naturelle est aussi le tremplin du sublime où la crainte et l'admiration se confondent dans une même représentation complexe de la Révolution. D'autres vertus argumentatives sont abordées. Notamment, la métaphore sert à distinguer les hommes sensibles et les ennemis de la vraie nature; tous les camps y recourent pour exclure leurs adversaires du débat. Parce qu'elle assimile la Révolution à des objets palpables ou à des phénomènes compréhensibles, la métaphore naturelle peut enfin définir les conditions de l'action politique, ce que Ritz illustre à travers le réseau d'images qui porte sur la navigation.

5 La deuxième partie, «Sciences et politique», montre comment la métaphore naturelle contribue à nouer et à dénouer les liens entre ces deux domaines. D'un côté, c'est en puisant dans le registre des naturalistes que des auteurs comme Volney, Condorcet ou Benjamin Constant tentent d'établir «une science naturelle des hommes en société» (p. 127). D'un autre côté, les métaphores d'un Chateaubriand trahissent bientôt la faillite d'une véritable science politique. Dans ces pages, Ritz suggère également que l'omniprésence des métaphores naturelles dans le discours politique influence en retour le débat scientifique, avant que la séparation des deux champs ne s'affirme.

6 La troisième partie, «Inventer la littérature», prolonge la question des champs disciplinaires aux belles-lettres. Les métaphores naturelles y sont abordées comme des «marqueurs littéraires» (p. 227) susceptibles de tracer des frontières entre les domaines et entre les professions. Ritz étudie d'abord la présence de métaphores naturelles, parfois empruntées au registre épique, dans les nombreuses histoires de la Révolution. Sous couvert d'impartialité, l'historien doit-il et peut-il même se passer de la métaphore, ou simplement d'une écriture passionnée, pour raconter des événements hors du commun? Ces questions se posent à travers l'œuvre riche en tensions de François Emmanuel Toulongeon, à laquelle Ritz consacre tout un chapitre. Dans la mesure où elle cristallise l'interconnexion des sciences, de la politique et de la 
littérature, la métaphore naturelle compromet le mouvement d'autonomisation de la littérature au début du XIX ${ }^{e}$ siècle et, à ce titre, elle peut nuire directement à la réception des œuvres qui se complaisent à l'employer. Finalement, Ritz remarque que la métaphore naturelle travaille aussi les textes de Staël, La Harpe et tous ceux qui écrivent sur la littérature. Celle-ci a besoin de se redéfinir après la "catastrophe» révolutionnaire. Les métaphores du vivant servent ainsi à représenter la littérature comme un organisme indépendant qui porte en lui «son propre principe de développement» (p. 316).

7 Les Métaphores naturelles dans le débat sur la Révolution manifestent une hygiène intellectuelle qui mérite d'être soulignée. D'une part, le chercheur reste proche de ses sources qu'il cite abondamment et qu'il ne réduit jamais aux positionnements idéologiques de leurs auteurs. D'autre part, en traquant la circulation de métaphores particulières dans des textes de genres distincts et d'obédiences politiques radicalement différentes, Ritz révèle d'un même geste la diversité et la forte cohérence d'un corpus problématique, souvent boudé dans les histoires littéraires.

8 Face à un travail de cette nature, on peut sans doute questionner la focalisation choisie. D'abord-et l'auteur en est bien conscient-la métaphore alimente un système d'images et de symboles qui engage d'autres procédés littéraires. Ensuite, le mot nature révèle une telle polysémie à la fin du xvIII ${ }^{\mathrm{e}}$ siècle qu'on se demande occasionnellement si toutes les métaphores naturelles appartiennent à un ensemble homogène. Là encore, Ritz apporte des réponses, par exemple en analysant ce qu'il appelle «le répertoire de la contre-nature» (p. 206). Enfin, certains lecteurs pourraient regretter que Ritz exclue de son enquête la poésie, un des lieux privilégiés du langage figuré. Les poèmes d'un Jacques Delille, par exemple, et les commentaires qu'ils suscitent, sont loin d'être étrangers au débat sur la Révolution. Surtout, ils jouent un rôle essentiel dans la reconfiguration d'une alliance entre les sciences et la littérature au tout début du XIX siècle.

9 Fin connaisseur de la Révolution, Ritz dispose d'une plume élégante, servie par un véritable sens de la formule. Son livre nous rappelle éloquemment que les écrits de la période révolutionnaire, malgré le poids de l'histoire et de la politique, ne déploient toutes leurs nuances, sinon leur ambivalence, qu'au prix d'une lecture littéraire, attentive aux enjeux esthétiques et rhétoriques. 